\title{
A CORAVEL radial-velocity monitoring of S stars: Symbiotic activity vs. orbital separation. III.*
}

\author{
J.M. Carquillat ${ }^{1}$, A. Jorissen ${ }^{2, \star \star}$, S. Udry ${ }^{3}$, and N. Ginestet ${ }^{1}$ \\ 1 Observatoire Midi-Pyrénées, UMR 5572, Av. E. Belin 14, F-31400 Toulouse, France \\ 2 Institut d'Astronomie et d'Astrophysique, Université Libre de Bruxelles, Campus Plaine, CP. 226, Bd. du Triomphe, B-1050 \\ Bruxelles, Belgium \\ 3 Observatoire de Genève, CH-1290 Sauverny, Switzerland
}

Received November 03, 1997; accepted January 29, 1998

\begin{abstract}
Orbital elements are presented for the Tc-poor S stars HR 363 (= HD 7351) and HD 191226. With an orbital period of $4592 \mathrm{~d}(=12.6 \mathrm{y})$, HR 363 has the longest period known among $\mathrm{S}$ stars, and yet it is a strong X-ray source. Its X-ray flux is similar to that of HD 35155, an S star with one of the shortest orbital periods (640 d). This surprising result is put in perspective with other diagnostics of binary interaction observed in binary $\mathrm{S}$ stars. They reveal that there is no correlation between the level of binary interaction and the orbital period. All these activity diagnostics moreover exhibit a strong time-variability. In the well-documented case of HR 1105, this time-variability appears to be a combination of orbital modulation and secular variation. A stream of gas from the red-giant wind, which is heated when funneled through the inner Lagrangian point, has been proposed as the source of the hard photons (Shcherbakov \& Tuominen 1992). Different viewing angles of the stream during the orbital cycle account for the orbital modulation, whereas long-term fluctuations of the mass-loss rate account for the secular variations. Little dependence to the orbital separation is expected for this kind of activity. If such streams are causing the activity observed in the other binary $\mathrm{S}$ stars as well, it would provide a natural explanation for the absence of correlation between orbital periods and activity levels, since the red-giant mass loss rate would be the dominant factor. The existence of such funneled streams is moreover predicted by smooth particle hydrodynamics simulations of mass transfer in detached binary systems.
\end{abstract}

Key words: stars: S — stars: HR 363, HD 191226 binaries: spectroscopic — binaries: symbiotic accretion — stars: late-type

Send offprint requests to: J.M. Carquillat

* Based on observations performed with the Swiss telescope at the Haute-Provence Observatory, France.

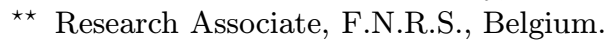

\section{Introduction}

This paper is the third one (see Udry et al. 1998a,b for the first two) in a series presenting new orbits obtained for barium and $\mathrm{S}$ stars as a result of a decade-long monitoring with the spectrovelocimeter CORAVEL (Baranne et al. 1979) installed on the 1-m Swiss telescope at the HauteProvence Observatory (OHP, France).

The two S stars HR 363 (=HD 7351) and HD 191226, that are the subject of this paper, were put on the CORAVEL monitoring program by both the Toulouse and the Geneva-Brussels teams, as part of larger samples. The Toulouse team aims at obtaining orbital elements for a sample of bright late-type stars quoted in the literature as being spectroscopic binaries, but with no orbit available. For the Geneva-Brussels team, HD 191226 and HR 363 belong to a sample of Tc-poor S stars, a family of chemicallypeculiar red giants suspected of being all binaries. A detailed discussion of the "binary paradigm" for Tc-poor S stars can be found in Jorissen et al. (1998), and needs not be repeated here.

The new orbits of HD 191226 and HR 363 are provided in Sects. 2 and 3, respectively. Diagnostics of binary interaction are available for these two systems from published observations in the UV and X domains. The availability of their orbital elements now offers the possibility to correlate these diagnostics of binary interaction with the orbital separation, as discussed in Sect. 4.

\section{HD 191226}

The spectroscopic binary nature of HD 191226 is already mentioned in the General Catalogue of Stellar Radial Velocities (GCSRV; Wilson 1953): from 10 measurements obtained at the Mount Wilson and Victoria observatories, the radial velocity of this gM2 star varied by $22 \mathrm{~km} \mathrm{~s}^{-1}$. Also classified as M2III by Nassau \& McRae (1949), whereas Barbier (1962) assigns it the spectral type K2II:, 
HD 191226 is finally recognized by Keenan \& Boeshaar (1980) as being a weak S star (M1S-M3SIIIa). The spectral class $\mathrm{MxS}$ refers to stars with the strongest $\mathrm{ZrO}$ bands barely visible. A spectrum obtained in the near infrared at a dispersion of $3.3 \mathrm{~nm} / \mathrm{mm}$ with the Aurélie spectrograph on the 1.52-m telescope at OHP yields a M0-M1III+ classification from a comparison with spectral standards (Ginestet et al. 1994; Carquillat et al. 1997). This spectrum is unfortunately of no use to confirm the S-type classification of HD 191226, as the typical S spectral features are better seen in the optical domain (Jaschek \& Jaschek 1987). The Tc-poor nature of HD 191226 was reported by Little et al. (1987) and Smith \& Lambert (1988).

The orbital elements of HD 191226 have been derived from the 36 radial-velocity measurements listed in Table 1. Among these, 31 were obtained with the CORAVEL spectrovelocimeter at OHP (with an average uncertainty of $\left.\bar{\epsilon}=0.34 \pm 0.06 \mathrm{~km} \mathrm{~s}^{-1}\right)$. One has been obtained at the Coudé focus of the 1.52-m telescope at OHP, on a baked IIaO plate (\# GA 8139), with a $2.0 \mathrm{~nm} / \mathrm{mm}$ dispersion. Four radial-velocity measurements obtained by Brown et al. (1990) on the 2.1-m telescope at McDonald Observatory equipped with a Reticon detector have also been used in the orbital solution. All these radial velocities, covering 3.37 orbital cycles, are on the system of IAU standards. The orbital elements listed in Table 2 have been computed with the BS1 program (Nadal et al. 1979) by assigning a weight 1 to the CORAVEL data and 0.25 to the five other measurements. The radial-velocity curve, folded with the orbital period, is presented in Fig. 1. Older data, obtained through the years 1912-1934, were not used in the orbital solution, because their accuracy is not good enough, even to improve the orbital period. The Mount Wilson data have (when available) uncertainties of the order of 2.5 to $3.3 \mathrm{~km} \mathrm{~s}^{-1}$, and their variation range ( -13 to $35 \mathrm{~km} \mathrm{~s}^{-1}$; Abt 1973) appears incompatible with the small value of the semi-amplitude $\left(K=4.8 \mathrm{~km} \mathrm{~s}^{-1}\right.$; Table 2$)$ derived from modern, more accurate measurements. As far as the the radial velocities obtained at Victoria by Harper (1934) are concerned, they are of doubtful quality (both plates are qualified as "weak") and were therefore not used either.

An estimate of the mass of the companion can be obtained from the mass function listed in Table 2. Adopting a mass of $1.5 \pm 0.5 M_{\odot}$ for the $\mathrm{S}$ star, which seems reasonable given its M2III spectral type (e.g., Schmidt-Kaler 1982; see also Jorissen et al. 1998), a minimum mass of $0.35 \pm 0.07 M_{\odot}$ for the secondary follows from the condition $\sin i \leq 1$. Taking $M_{1}=1.5 M_{\odot}$ for the red giant and $M_{2}=0.6 M_{\odot}$ for its companion yields $i=40^{\circ}$, $q=M_{2} / M_{1}=0.4$ and $A_{1}=121 \mathrm{Gm}$, or in terms of orbital separation, $A=A_{1}+A_{2}=A_{1}(1+1 / q)=2.8 \mathrm{AU}$.

The companion mass is thus compatible with that of a white dwarf (WD). Observations in the UV with the Goddard High Resolution Spectrograph (GHRS) on board the Hubble Space Telescope indeed reveal the presence of
Table 1. Radial velocities of HD 191226. Column $4\left(\epsilon_{1}\right)$ lists the uncertainty on the individual measurements. The columns labelled "Phase" and "O-C" refer to the orbital solution listed in Table 2. The column labelled "Obs" provides the origin of the radial velocity: COR $=$ CORAVEL; Cou $=$ Coudé spectrum obtained at OHP; McD = data obtained by Brown et al. (1990) at McDonald observatory

\begin{tabular}{lccccc}
\hline HJD & Phase & $\begin{array}{c}\text { RV } \\
\left(\mathrm{km} \mathrm{s}^{-1}\right)\end{array}$ & $\begin{array}{c}\epsilon_{1} \\
\left(\mathrm{~km} \mathrm{~s}^{-1}\right)\end{array}$ & $\begin{array}{c}\mathrm{O}-\mathrm{C} \\
\left(\mathrm{km} \mathrm{s}^{-1}\right)\end{array}$ & Obs \\
\hline 46337.343 & 0.229 & -22.68 & 0.32 & 0.0 & COR \\
46587.548 & 0.436 & -21.16 & 0.30 & 0.0 & COR \\
46719.370 & 0.544 & -22.20 & 0.34 & -0.1 & COR \\
46965.534 & 0.748 & -26.68 & 0.48 & -0.4 & COR \\
47099.324 & 0.858 & -28.86 & 0.36 & +0.4 & COR \\
47100.360 & 0.859 & -29.94 & 0.40 & -0.6 & COR \\
47285.598 & 0.012 & -29.86 & 0.30 & -0.1 & COR \\
47343.000 & 0.060 & -28.7 & 0.7 & -0.4 & McD \\
47369.000 & 0.081 & -28.6 & 0.7 & -1.1 & McD \\
47397.462 & 0.105 & -26.0 & 0.9 & +0.6 & Cou \\
47460.291 & 0.157 & -24.81 & 0.33 & -0.1 & COR \\
47497.000 & 0.187 & -24.5 & 0.7 & -0.8 & McD \\
47519.000 & 0.205 & -22.7 & 0.7 & +0.5 & McD \\
47868.264 & 0.494 & -22.15 & 0.30 & -0.5 & COR \\
48128.435 & 0.709 & -24.64 & 0.32 & +0.7 & COR \\
48137.444 & 0.716 & -25.06 & 0.29 & +0.4 & COR \\
48936.362 & 0.376 & -21.54 & 0.32 & -0.4 & COR \\
49100.589 & 0.512 & -21.77 & 0.29 & 0.0 & COR \\
49145.572 & 0.549 & -22.65 & 0.31 & -0.4 & COR \\
49185.575 & 0.582 & -23.39 & 0.30 & -0.7 & COR \\
49250.322 & 0.636 & -24.18 & 0.29 & -0.6 & COR \\
49319.277 & 0.693 & -25.15 & 0.29 & -0.2 & COR \\
49482.565 & 0.827 & -28.48 & 0.31 & 0.0 & COR \\
49546.501 & 0.880 & -29.81 & 0.31 & 0.0 & COR \\
49639.309 & 0.957 & -30.47 & 0.29 & +0.1 & COR \\
49640.402 & 0.958 & -31.03 & 0.30 & -0.4 & COR \\
49643.329 & 0.960 & -30.24 & 0.29 & +0.4 & COR \\
49783.716 & 0.076 & -27.37 & 0.32 & +0.3 & COR \\
49899.537 & 0.172 & -24.31 & 0.30 & -0.1 & COR \\
49959.354 & 0.221 & -22.45 & 0.33 & +0.4 & COR \\
50194.611 & 0.416 & -21.08 & 0.31 & 0.0 & COR \\
50314.419 & 0.515 & -21.33 & 0.29 & +0.5 & COR \\
50329.496 & 0.527 & -21.63 & 0.30 & +0.3 & COR \\
50359.335 & 0.552 & -21.42 & 0.29 & +0.8 & COR \\
50382.257 & 0.571 & -22.21 & 0.31 & +0.3 & COR \\
50416.295 & 0.599 & -22.81 & 0.40 & +0.1 & COR \\
\hline & & & & &
\end{tabular}

Table 2. Orbital elements of HD 191226 and HR 363

\begin{tabular}{lllll}
\hline & \multicolumn{2}{c}{ HD 191226} & \multicolumn{2}{c}{ HR 363 } \\
\hline$P(\mathrm{~d})$ & 1210.4 & \pm 4.3 & 4592.7 & \pm 110.0 \\
$T_{0}$ & $2448481 \pm 24$ & 2444 & $696 \pm 230$ \\
$e$ & 0.19 & \pm 0.02 & 0.17 & \pm 0.03 \\
$V_{0}\left(\mathrm{~km} \mathrm{~s}^{-1}\right)$ & -25.05 & \pm 0.08 & 1.55 & \pm 0.15 \\
$\omega\left(^{\circ}\right)$ & 207.4 & \pm 7.5 & 104.3 & \pm 13.7 \\
$K\left(\mathrm{~km} \mathrm{~s}^{-1}\right)$ & 4.76 & \pm 0.11 & 5.43 & \pm 0.16 \\
$A_{1} \sin i(\mathrm{Gm})$ & 77.76 & \pm 1.85 & 337.5 & \pm 12.9 \\
$f(M)\left(M_{\odot}\right)$ & 0.0128 & \pm 0.0009 & 0.073 & \pm 0.007 \\
$\sigma(\mathrm{O}-\mathrm{C})\left(\mathrm{km} \mathrm{s}^{-1}\right)$ & \multicolumn{2}{c}{0.38} & \multicolumn{2}{c}{0.68} \\
$N$ & \multicolumn{2}{c}{36} & \multicolumn{2}{c}{50} \\
\hline
\end{tabular}




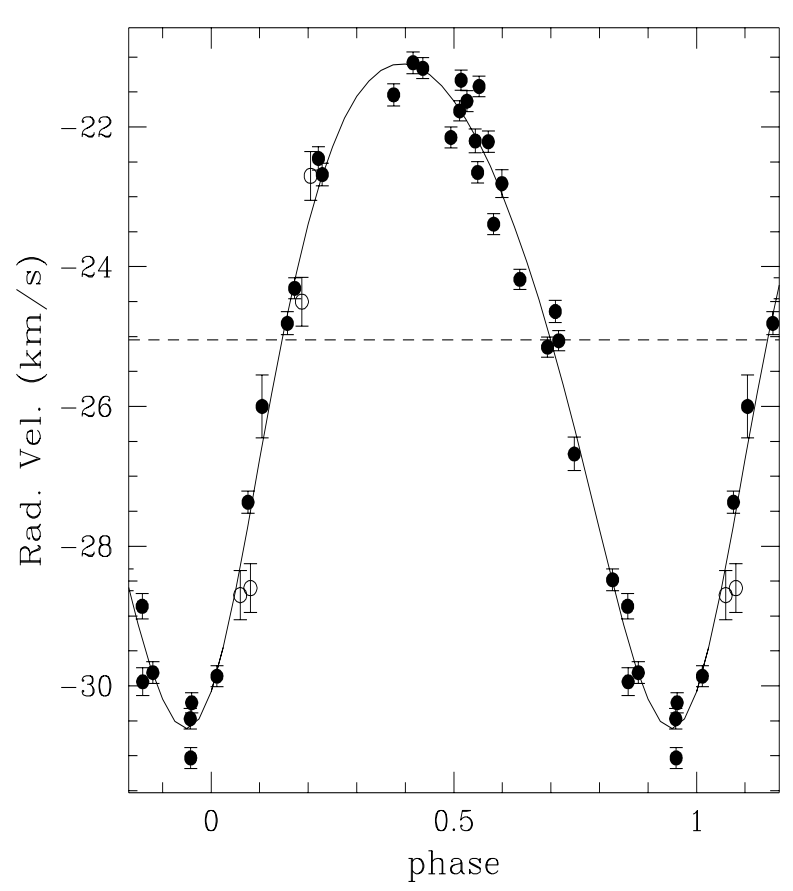

Fig. 1. The radial-velocity curve of HD 191226, folded with the orbital period. Filled dots refer to OHP data (CORAVEL and Coudé), and open dots to McDonald data

a WD with $T \sim 15000 \mathrm{~K}$ (Ake 1997). These results will be discussed further in Sect. 4 .

\section{HR 363}

As for HD 191226, the spectroscopic-binary nature of HR 363 (= HD 7351) is already mentioned in the GCSRV, where it is classified as gM2. Six radial-velocity measurements are mentioned in that catalogue, among which 4 were performed at the David Dunlap Observatory (Young 1945) and 2 at the Mount Wilson Observatory (Wilson \& Joy 1952; Abt 1970). These two sets of measurements yield average velocities of +5.8 and $-1.8 \mathrm{~km} \mathrm{~s}^{-1}$, respectively. It is likely that it is this difference which led to suspect the binary nature of HR 363 in the GCSRV. More recently, three radial-velocity measurements were obtained at the E.W. Fick Observatory (Beavers \& Eitter 1986), and 15 more by Brown et al. (1990). The latter measurements, covering $1000 \mathrm{~d}$, confirmed the spectroscopic-binary nature of HR 363, and made it clear that its period was quite long.

HR 363 was classified M2S by Keenan (1954), who noted its strong BaII $\lambda 455.4 \mathrm{~nm}$ line, and M3IIS by Yamashita (1967). Keenan \& Boeshaar (1980) reclassified it later on as $\mathrm{S} 3+/ 2-$ in their revised classification scheme (where the first digit is a temperature index, and the second one is an index of $\mathrm{ZrO}$ strength, 2 corresponding to $\mathrm{ZrO}<\mathrm{TiO}$ ). More recently, Sato \& Kuji (1990) have

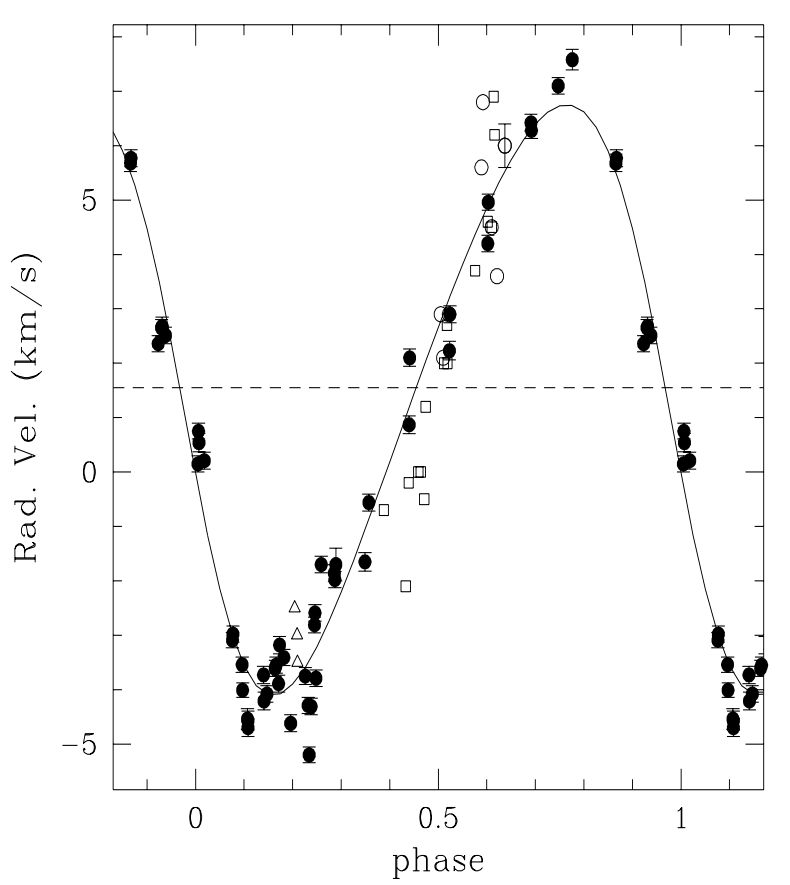

Fig. 2. The radial-velocity curve of HR 363 (=HD 7351), folded with the orbital period. Filled dots refer to CORAVEL data, and open circles to radial velocities derived from photographic spectra obtained at the Haute-Provence Observatory $(1.52-\mathrm{m}$ telescope). Measurements from Brown et al. (1990) and from Beavers \& Eitter (1976) are represented by open squares and open triangles, respectively. Only the CORAVEL data, plus one OHP photographic measurement (open circle with an error bar), have been used to derive the orbital solution

classified HR 363 as M2III. These authors stress that, although HR 363 is often considered as an S star, its salient spectral features are those typical of $\mathrm{M}$ giants, except for a strong BaII $\lambda 455.4 \mathrm{~nm}$ line. As for HD 191226, a nearinfrared spectrum has been obtained for HR 363 with the Aurélie spectrograph at OHP, and yields a M3III spectral type. Finally, regarding its Tc-poor nature, we refer to Little et al. (1987) and Smith \& Lambert (1988).

The orbital solution for HR 363 listed in Table 2 is based on 49 CORAVEL radial-velocity measurements covering 1.1 orbital cycle (from 1983 to 1997), to which one older measurement obtained in 1976 has been added (Table 3). This early measurement (obtained at the 1.52-m telescope of Haute-Provence Observatory on the photographic spectrum GA 2881) substantially improves the period determination, since it brings the orbital coverage to 1.65 cycle. Its accuracy is only $0.8 \mathrm{~km} \mathrm{~s}^{-1}$, compared to an average of $0.30 \mathrm{~km} \mathrm{~s}^{-1}$ for the CORAVEL measurements. It has therefore been attributed a weight of 0.25 in the orbital solution (compared to 1 for the CORAVEL measurements).

The radial-velocity curve, folded with the orbital period, is presented in Fig. 2. This figure presents as well 
Table 3. Radial velocities of HR $363=$ HD 7351. Symbols are as in Table 1

\begin{tabular}{|c|c|c|c|c|c|}
\hline $\begin{array}{l}\text { HJD } \\
2400000+\end{array}$ & Phase & $\begin{array}{c}\mathrm{RV} \\
\left(\mathrm{km} \mathrm{s}^{-1}\right)\end{array}$ & $\begin{array}{c}\epsilon_{1} \\
\left(\mathrm{~km} \mathrm{~s}^{-1}\right)\end{array}$ & $\begin{array}{c}\mathrm{O}-\mathrm{C} \\
\left(\mathrm{km} \mathrm{s}^{-1}\right)\end{array}$ & Obs \\
\hline 43026.6290 & 0.637 & +6.0 & 0.8 & +0.5 & $\mathrm{Cou}$ \\
\hline 45340.3280 & 0.140 & -3.73 & 0.32 & +0.4 & $\mathrm{COR}$ \\
\hline 45343.2657 & 0.141 & -4.21 & 0.33 & -0.1 & $\mathrm{COR}$ \\
\hline 45595.6070 & 0.196 & -4.62 & 0.31 & -0.7 & $\mathrm{COR}$ \\
\hline 46008.4922 & 0.286 & -1.86 & 0.31 & +0.6 & $\mathrm{COR}$ \\
\hline 46015.4520 & 0.287 & -1.98 & 0.30 & +0.5 & $\mathrm{COR}$ \\
\hline 46296.5681 & 0.349 & -1.65 & 0.34 & -0.5 & $\mathrm{COR}$ \\
\hline 46335.5978 & 0.357 & -0.56 & 0.31 & +0.3 & $\mathrm{COR}$ \\
\hline 46715.5295 & 0.440 & +0.87 & 0.33 & -0.3 & $\mathrm{COR}$ \\
\hline 46722.5150 & 0.441 & +2.10 & 0.32 & +0.9 & $\mathrm{COR}$ \\
\hline 47097.4289 & 0.523 & +2.23 & 0.34 & -1.0 & $\mathrm{COR}$ \\
\hline 47101.4738 & 0.524 & +2.90 & 0.31 & -0.3 & $\mathrm{COR}$ \\
\hline 47459.4887 & 0.602 & +4.20 & 0.31 & -0.7 & $\mathrm{COR}$ \\
\hline 47463.4908 & 0.603 & +4.96 & 0.30 & +0.1 & $\mathrm{COR}$ \\
\hline 47867.3641 & 0.691 & +6.42 & 0.31 & +0.1 & $\mathrm{COR}$ \\
\hline 47872.3239 & 0.692 & +6.28 & 0.30 & +0.0 & $\mathrm{COR}$ \\
\hline 48128.5884 & 0.747 & +7.10 & 0.30 & +0.4 & $\mathrm{COR}$ \\
\hline 48261.3514 & 0.776 & +7.58 & 0.38 & +0.9 & $\mathrm{COR}$ \\
\hline 48671.2717 & 0.866 & +5.68 & 0.32 & +0.2 & $\mathrm{COR}$ \\
\hline 48676.2604 & 0.867 & +5.77 & 0.31 & +0.3 & $\mathrm{COR}$ \\
\hline 48936.5273 & 0.923 & +2.36 & 0.30 & -1.2 & $\mathrm{COR}$ \\
\hline 48967.3589 & 0.930 & +2.65 & 0.30 & -0.7 & $\mathrm{COR}$ \\
\hline 48972.4352 & 0.931 & +2.68 & 0.34 & -0.5 & $\mathrm{COR}$ \\
\hline 49002.2925 & 0.938 & +2.51 & 0.30 & -0.5 & $\mathrm{COR}$ \\
\hline 49313.4231 & 0.005 & +0.15 & 0.30 & +0.5 & $\mathrm{COR}$ \\
\hline 49317.3760 & 0.006 & +0.75 & 0.29 & +1.0 & $\mathrm{COR}$ \\
\hline 49321.4032 & 0.007 & +0.54 & 0.34 & +0.8 & $\mathrm{COR}$ \\
\hline 49371.2581 & 0.018 & +0.21 & 0.31 & +1.1 & $\mathrm{COR}$ \\
\hline 49639.6748 & 0.076 & -3.09 & 0.28 & -0.1 & $\mathrm{COR}$ \\
\hline 49640.5580 & 0.077 & -2.98 & 0.30 & +0.0 & $\mathrm{COR}$ \\
\hline 49730.2663 & 0.096 & -3.54 & 0.28 & +0.0 & $\mathrm{COR}$ \\
\hline 49734.2638 & 0.097 & -4.01 & 0.27 & -0.5 & $\mathrm{COR}$ \\
\hline 49781.2730 & 0.107 & -4.54 & 0.38 & -0.8 & $\mathrm{COR}$ \\
\hline 49783.2658 & 0.108 & -4.70 & 0.32 & -1.0 & $\mathrm{COR}$ \\
\hline 49785.2729 & 0.108 & -4.57 & 0.35 & -0.9 & $\mathrm{COR}$ \\
\hline 49964.6007 & 0.147 & -4.08 & 0.31 & +0.0 & $\mathrm{COR}$ \\
\hline 50042.3732 & 0.164 & -3.61 & 0.30 & +0.5 & $\mathrm{COR}$ \\
\hline 50052.3666 & 0.166 & -3.55 & 0.29 & +0.6 & $\mathrm{COR}$ \\
\hline 50072.2763 & 0.171 & -3.89 & 0.31 & +0.2 & $\mathrm{COR}$ \\
\hline 50083.3657 & 0.173 & -3.18 & 0.32 & +0.9 & $\mathrm{COR}$ \\
\hline 50123.2461 & 0.182 & -3.41 & 0.30 & +0.6 & $\mathrm{COR}$ \\
\hline 50325.5841 & 0.226 & -3.75 & 0.31 & -0.2 & $\mathrm{COR}$ \\
\hline 50355.4486 & 0.232 & -4.29 & 0.30 & -0.8 & $\mathrm{COR}$ \\
\hline 50363.4453 & 0.234 & -5.20 & 0.29 & -1.7 & $\mathrm{COR}$ \\
\hline 50382.3557 & 0.238 & -4.31 & 0.30 & -0.9 & $\mathrm{COR}$ \\
\hline 50415.4728 & 0.245 & -2.81 & 0.29 & +0.5 & $\mathrm{COR}$ \\
\hline 50420.3984 & 0.246 & -2.59 & 0.31 & +0.7 & $\mathrm{COR}$ \\
\hline 50428.3299 & 0.248 & -3.79 & 0.30 & -0.6 & $\mathrm{COR}$ \\
\hline 50476.3083 & 0.259 & -1.70 & 0.30 & +1.3 & $\mathrm{COR}$ \\
\hline 50615.6170 & 0.289 & -1.7 & 0.30 & +0.7 & $\mathrm{COR}$ \\
\hline
\end{tabular}

the other measurements (namely the three radial velocities from the Fick Observatory, the 15 measurements from Brown et al., and the 6 radial velocities we obtained from photographic spectra at OHP) that were not used in the orbital solution, since they would degrade its accuracy. These observations are nevertheless compatible with the computed solution. Note that the $\mathrm{O}-\mathrm{C}$ residuals are significantly larger than the accuracy of the measurements. This jitter is likely due to envelope pulsations or to atmospheric motions, as discussed by Jorissen et al. (1998; see their Fig. 1).

As for HD 191226, the mass function is compatible with a WD companion, since $M_{1}=1.5 \pm 0.5 M_{\odot}$ for the red giant implies $M_{2}>0.70 \pm 0.15 M_{\odot}$ for the unseen companion, given $\sin i \leq 1.0$. If the companion is to be a WD with a mass typical of field WD's $\left(0.58 M_{\odot}\right.$; Reid 1996), the orbital inclination has to be close to $90^{\circ}$, and the system may be an eclipsing binary.

This star has been observed with the International Ultraviolet Explorer (IUE) and ROSAT satellites. Although there is only marginal evidence for an UV continuum from a hot companion (Ake et al. 1988), HR 363 is a strong source of hard X-rays (Jorissen et al. 1996). These $\mathrm{X}$-rays are not expected to come from a hot corona, because with $B-V=1.7$, HR 363 lies far to the right of the region of the Hertzsprung-Russell diagram populated by class III giants with a hot corona (Hünsch et al. 1996). The hard X-rays in HR 363 are therefore likely powered by mass transfer in the binary system. The same holds true for the weak HeI $\lambda 1083.0 \mathrm{~nm}$ emission line observed in HR 363 (Brown et al. 1990), since that line is generally absent in cool $\mathrm{M}$ giants, but is frequent in interacting binary systems like symbiotic stars (Brown et al. 1990 and references therein). It is somewhat surprising, though, that HR 363 behaves as an interacting binary system while having the longest known period (4593 d = $12.6 \mathrm{y}$ ) among $\mathrm{S}$ stars (Jorissen et al. 1998). That question is discussed in more details in Sect. 4.

\section{Binary interaction vs. orbital separation}

Binary S stars share many properties with symbiotic stars, since both families consist of a cool red giant and a WD companion in systems with orbital periods of a few hundred to a few thousand days (Jorissen 1997). Some kind of symbiotic activity should thus be expected among binary S stars as well. Table 4 lists those systems where the usual signatures of binary interaction have been probed. In that table, $L_{\mathrm{X}}$ refers to the luminosity in the ROSAT hard band $(0.5-2.4 \mathrm{keV})$ taken from Jorissen et al. (1996), and adapted to the new HIPPARCOS distances (Van Eck et al. 1998). Hard X-rays have been observed in HR 363 and HD 35155, and appear strongly variable. In the UV domain, several stars exhibit strong emission lines of highly ionized species typical of interacting binary systems (like 
Table 4. Signatures of binary interaction in extrinsic S stars, arranged in order of increasing orbital period. The orbital separations at apastron and periastron ( $A_{\text {apa }}$ and $A_{\text {peri }}$, respectively) have been computed from Kepler's third law assuming a total mass of $2 M_{\odot}$ for all systems. In column "UV emission lines": "no" = no emission lines detected; "IB" = emission lines typical of interacting binaries; "WD" = WD continuum

\begin{tabular}{|c|c|c|c|c|c|c|c|c|c|c|c|}
\hline \multirow[t]{2}{*}{ Name } & \multirow[t]{2}{*}{ Sp. Typ. } & \multirow{2}{*}{$\begin{array}{r}P \\
(\mathrm{~d}) \\
\end{array}$} & \multirow[t]{2}{*}{$e$} & \multirow{2}{*}{$\begin{array}{c}A_{\text {peri }}-A_{\text {apa }} \\
(\mathrm{AU})\end{array}$} & \multirow{2}{*}{$M_{\mathrm{bol}}$} & \multirow{2}{*}{\multicolumn{2}{|c|}{$\begin{array}{c}L_{\mathrm{X}} \\
\left(L_{\odot}\right) \\
\end{array}$}} & \multicolumn{2}{|c|}{ UV } & \multirow{2}{*}{$\begin{array}{l}\mathrm{H}_{\alpha} \\
\mathrm{em} .\end{array}$} & \multirow{2}{*}{$\begin{array}{c}\mathrm{HeI} \\
\lambda 1083.0 \mathrm{~nm} \\
\end{array}$} \\
\hline & & & & & & & & $L_{\mathrm{SWP}} / L_{\odot}$ & em. lines & & \\
\hline HD 121447 & K4IIIBa4.5 & 185.7 & 0.0 & $0.8-0.8$ & -1.4 & & $?$ & $<0.002$ & no & $?$ & $?$ \\
\hline HR 1105 & $\mathrm{~S} 3.5 / 2$ & 596.2 & $0.09 \pm 0.02$ & $1.6-1.9$ & -3.1 & & $?$ & $0.003-0.02$ & IB & $?$ & var \\
\hline HD 35155 & $\mathrm{~S} 3 / 2$ & 640.5 & $0.07 \pm 0.02$ & $1.7-2.0$ & $-3.1^{z}$ & $<6(-4)$ & 4) $-1(-3)^{x}$ & $0.08-0.2$ & IB & $\mathrm{y}$ & var \\
\hline HD 191226 & M1-3S & 1210.4 & $0.19 \pm 0.02$ & $2.3-3.3$ & $-{ }^{z}$ & & $?$ & - & WD & $?$ & wk em \\
\hline HD $49368^{y}$ & $\mathrm{~S} 3 / 2$ & 2995.9 & $0.36 \pm 0.05$ & $3.3-7.0$ & -3.4 & & $?$ & 0.005 & IB & $?$ & abs \\
\hline HR 363 & $\mathrm{~S} 3 / 2$ & 4592.7 & $0.17 \pm 0.03$ & $5.7-8.0$ & -3.2 & $<7(-5)$ & $-1.5(-3)$ & 0.002 & no & $?$ & wk em \\
\hline Ref & $\mathrm{a}$ & $\mathrm{b}$ & $\mathrm{b}$ & $\mathrm{b}$ & $\mathrm{c}$ & & $\mathrm{d}$ & $\mathrm{e}$ & $\mathrm{f}$ & $\mathrm{g}$ & $\mathrm{h}$ \\
\hline
\end{tabular}

References: a: Keenan \& Boeshaar (1980), Keenan \& McNeil (1989); b: Jorissen et al. (1998); c: Van Eck et al. (1998); d: Jorissen et al. (1996); e: Flux densities at $160 \mathrm{~nm}$ from Johnson et al. (1993) combined with distances from Van Eck et al. (1998) or Eggen (1972); f: Johnson et al. (1993), Ake (1997); g: Ake et al. (1991); h: Brown et al. (1990), Shcherbakov \& Tuominen (1992).

Remarks: x: 1(-3) stands for $110^{-3}$; y: HD 49368 = V613 Mon; z: HIPPARCOS parallax very uncertain; for HD 35155, distance from Eggen (1972) adopted instead.

CIV $\lambda 155.0 \mathrm{~nm})$. In the column labelled "UV em. lines", "IB" stands for "interacting binary", "no" stands for no emission lines seen, and "WD" indicates that the UV spectrum fits that of a clean WD. The continuum UV luminosity in the $125.0-195.0 \mathrm{~nm}$ band is often larger than would be expected from an isolated WD [see column " $L_{\mathrm{SWP}}$ "]. In Table $4, L_{\mathrm{SWP}}=4 \pi d^{2} 70 f(160 \mathrm{~nm})$, where the average flux density $f(160 \mathrm{~nm})$ in the $125.0-195.0$ $\mathrm{nm}$ domain is taken from Johnson et al. (1993) and the distance $d$ from Van Eck et al. (1998) or Eggen (1972). The UV luminosity is often strongly variable, in which case the different observed values are listed. The HeI $\lambda$ $1083.0 \mathrm{~nm}$ triplet generally confirms the UV diagnostics. The three binary S stars flagged as "interacting binaries" from their UV features are also those showing strong and variable HeI $\lambda 1083.0 \mathrm{~nm}$ lines, whereas the two stars (HR 363 and HD 191226) with no UV emission lines exhibit only a weak HeI triplet in emission.

Two important conclusions may be drawn from the observations summarized in Table 4:

(i) the level of binary interaction does not appear to be correlated with the orbital period. HD 49368 (=V613 Mon) for instance exhibits a much higher level of activity than the shorter-period system HD 191226, whereas the shortestperiod system HD 121447 does not show any sign of interaction at all. Moreover, the maximum X-ray luminosities of HR 363 and HD 35155 are comparable despite very different orbital periods;

(ii) the activity appears to be strongly variable.

Orbital modulation is likely a major cause of the activity variations, as shown by Shcherbakov \& Tuominen (1992) and Ake et al. (1994) in the well-documented case of HR 1105. However, at a given phase, important cycle- to-cycle variations remain (Ake et al. observed a variation by a factor of $\sim 3$ in the $\lambda 146.0 \mathrm{~nm}$ flux of HR 1105 at phase 0.3 in two different orbital cycles), suggesting the existence of yet another cause of (secular) variability.

Various physical processes are able to produce hard photons modulated by the orbital motion in a binary system:

1. Heating of the red-giant hemisphere facing a hot WD;

2. Accretion-powered hot spot;

3. Stream of gas from the red-giant wind, heated when funneled through the inner Lagrangian point.

A strong sensitivity of the activity level to the binary separation is expected in the first and second cases. In the first case, the orbital separation directly controls the dilution suffered by the hot radiation when it reaches the giant atmosphere. In the second case, the mass accretion rate by the secondary roughly scales as $\dot{M} k^{4} /\left(1+k^{2}\right)^{3 / 2}$ (where $\dot{M}$ is the wind mass-loss rate of the giant, and $k$ is the ratio between the orbital and the wind velocities) in the case of supersonic Bondi-Hoyle accretion in a detached system (see Theuns et al. 1996). The previous relation reduces to $\dot{M} A^{-2}$ (where $A$ is the orbital separation) when $k<<1$ (i.e. $v_{\text {wind }}>>v_{\text {orb }}$ ).

From a detailed analysis of the variations with orbital phase of the UV flux level and the HeI $\lambda 1083.0 \mathrm{~nm}$ line shape, Shcherbakov \& Tuominen (1992) and Ake et al. (1994) favour the third process as the origin of the hard photons, i.e. funneling of the red-giant wind through the inner Lagrangian point. The existence of such funneled streams is moreover predicted by smooth particle hydrodynamics simulations of mass transfer in detached binary systems (Theuns \& Jorissen 1993; Theuns et al. 1996). Different viewing angles of the stream during the orbital 
cycle account for the orbital modulation, whereas longterm fluctuations of the mass-loss rate account for the secular variations (like those observed in Mira variables, and associated with a clumpy and non-spherically symmetric wind; e.g. Whitelock et al. 1997; Lopez et al. 1997; Olofsson 1997). The wind mass-loss rate of the red giant, rather than the orbital separation, is expected to be the dominant factor controlling the activity level in this case. The absence of any correlation between the orbital periods and the activity levels in the sample of $\mathrm{S}$ stars listed in Table 4 therefore suggests that streams like the one observed in the system HR 1105 might in fact be responsible for the activity observed in other $\mathrm{S}$ stars as well. The absence of any activity observed in the system HD 121447, despite the fact that it is the closest system in the sample, may then be attributed to its low luminosity $\left(M_{\mathrm{bol}}=-1.4\right)$, and therefore low mass-loss rate. Among the more luminous $\mathrm{S}$ stars $\left(M_{\mathrm{bol}} \sim-3.2\right)$, differences in their mass-loss rates may account for their different activity levels (compare e.g. HD 35155 and HR 1105 having different activity levels despite similar periods and spectral types, or HD 35155 and HR 363 having the same $\mathrm{X}$-ray flux at very different orbital periods).

Future detailed studies of this class of mass-losing, binary red giants may thus be expected to shed light on the mass-loss process, as well as on the physics of interacting binaries.

Acknowledgements. We wish to express our thanks to Tom Ake for communicating us results in advance of publication. Data and bibliographic references made available by the Centre de Données Stellaires (Strasbourg) were of great help in the present study. This work was supported in part by the Fonds National de la Recherche Scientifique (Belgium, Switzerland).

\section{References}

Abt H.A., 1970, ApJS 19, 387

Abt H.A., 1973, ApJS 26, 472

Ake T.B., 1997, in: Wing R.F. (ed.) The Carbon Star Phenomenon (IAU Symp. 177). Kluwer, Dordrecht (in press)

Ake T.B., Johnson H.R., Peery B.F.Jr., 1988, in: Rolfe E.J. (ed.) A Decade of UV Astronomy with IUE. ESA-SP 281, p. 245

Ake T.B., Johnson H.R., Ameen M.M., 1991, ApJ 383, 842

Ake T.B., Johnson H.R., Bopp B.W., 1994, in: Shafter A.W. (ed.) Interacting Binary Stars. ASP Conf. Ser. 56, p. 413

Baranne A., Mayor M., Poncet J.L., 1979, Vistas Astron. 23, 279

Barbier M., 1962, J. Obs. 45, 57

Beavers W.I., Eitter J.J., 1986, ApJS 62, 147
Brown J.A., Smith V.V., Lambert D.L., Dutchover E.Jr., Hinkle K.H., Johnson H.R., 1990, AJ 99, 1930

Carquillat J.M., Jaschek C., Jaschek M., Ginestet N., 1997, A\&AS 123,5

Eggen O.J., 1972, ApJ 177, 489

Ginestet N., Carquillat J.M., Jaschek M., Jaschek C., 1994, A\&AS 108, 359

Harper W.E., 1934, Publ. Dominion Astrophys. Obs. 6, 151

Hünsch M., Schmitt J.H.M.M., Schröder K.-P., Reimers D., 1996, A\&A 310, 801

Jaschek C., Jaschek M., 1987, The Classification of Stars. Cambridge Univ. Press

Johnson H.R., Ake T.B., Ameen M.M., 1993, ApJ 402, 667

Jorissen A., 1997, in: Mikolajewska J. (ed.) Physical Processes in Symbiotic Stars and Related Systems. Copernicus Found. for Polish Astronomy, Warsaw, p. 135

Jorissen A., Schmitt J.H.M.M., Carquillat J.M., Ginestet N., Bickert K.F., 1996, A\&A 306, 467

Jorissen A., Van Eck S., Mayor M., Udry S., 1998, A\&A (in press)

Keenan P.C., 1954, ApJ 120, 484

Keenan P.C., Boeshaar P.C., 1980, ApJS 43, 379

Keenan P.C., McNeil R.C., 1989, ApJS 71, 245

Little, S.J., Little-Marenin, I.R., Hagen-Bauer, W., 1987, AJ 94, 981

Lopez B., Danchi W.C., Bester M., et al., 1997, ApJ 488, 807

Nadal R., Ginestet N., Carquillat J.M., Pédoussaut A., 1979, A\&AS 35, 203

Nassau J.J., McRae D.A., 1949, ApJ 110, 478

Olofsson H., 1997, in: Wing R. (ed.) The Carbon Star Phenomenon (IAU Symp. 177). Kluwer, Dordrecht (in press)

Reid I.N., 1996, AJ 111, 2000

Sato K., Kuji S., 1990, A\&AS 85, 1069

Schmidt-Kaler Th., 1982, in: Landolt-Börnstein (ed.) Zahlenwerte und Funktionen aus Naturwissenschaften und Technik, Group 6, Vol. 2. Springer-Verlag, Berlin, p. 1

Shcherbakov A.G., Tuominen I., 1992, A\&A 255, 215

Smith V.V., Lambert D.L., 1988, ApJ 333, 219

Theuns T., Jorissen A., 1993, MNRAS 265, 946

Theuns T., Boffin H.M.J., Jorissen A., 1996, MNRAS 280, 1264

Udry S., Mayor M., Van Eck S., Jorissen A., 1998a, A\&AS 131, 25

Udry S., Mayor M., Van Eck S., Jorissen A., Prévot L., Grenier S., Lindgren H., 1998b, A\&AS 131, 43

Van Eck S., Jorissen A., Udry S., Mayor M., Pernier B., 1998, A\&A 329, 971

Whitelock P.A., Feast M.W., Marang F., Overbeek M.D., 1997, MNRAS 288, 512

Wilson R.E., 1953, General Catalogue of Stellar Radial Velocities, Carnegie Inst. Washington Publ. 601 (GCSRV)

Wilson R.E., Joy A.H., 1952, ApJ 115, 157

Yamashita Y., 1967, Publ. DAO 13, 47

Young R.K., 1945, Publ. DDO 1, 311 